\title{
Outcome of Band Ligation in Esophageal Varices of Bangladeshi Children: A Tertiary Centre Experience
}

\author{
SALAHUDDIN MAHMUD ${ }^{1}$, SYED SHAFI AHMED ${ }^{2}$, JAHIDA GULSHAN ${ }^{3}$, \\ FARHANA TASNEEM ${ }^{4}$, MADHABI BAIDYA ${ }^{5}$
}

\begin{abstract}
Background: Variceal bleeding is often a life threatening clinical situation in infants and children. Band ligation is the main endoscopic treatment for esophageal varices.

Objective: To see the outcome of band ligation of esophageal varices in extra-hepatic and hepatic cases of portal hypertension.

Methods: This prospective study was done in the Department of Pediatric Gastroenterology, Hepatology \& Nutrition, Dhaka Shishu (Children) Hospital, Dhaka, Bangladesh on 40 consecutive cases of esophageal varices enrolled from April, 2014 to March 2016. Every case was treated with band ligation followed by tab. propranolol. Cases were followed up for a minimum period of one year after the band ligation.

Results: Age of the children was 2-12 years with mean age of $7.2 \pm 4.3$ years and male:female ratio was 1.5:1. Out of 40 children, 32 (80\%) were pre-hepatic and 8 (20\%) hepatic (chronic liver disease with portal hypertension) causes. Only 1 session required in 50\% pre-hepatic cases and multiple (2-3) sessions required in hepatic $(100 \%)$ cases. Almost same number of band (average 2-3) required in every session of both cases. Grade-ll esophageal varices with red sign were more common in prehepatic cases \& severity of grading much more (grade-III \& IV) in hepatic cases. Gastric varices were more common in hepatic (50\%) cases than extra-hepatic $(12.5 \%)$ cases. Recurrence of bleeding occurred in all hepatic (100\%) cases and half (50\%) of the pre-hepatic cases. Early re-bleeding was more common in hepatic $(75 \%)$ cases \& late re-bleeding in both pre-hepatic (43.7\%) \& hepatic (100\%) cases. Minimal side effect like discomfort (10\%) \& Nausea (10\%) were present after the procedure.

Conclusion: Pre-hepatic was the most common etiology of portal hypertension in studied children. Fewer sessions were required in pre-hepatic cases than in hepatic cases. Severity of grading, re-bleeding \& associated gastric varices were more common in hepatic cases. Band ligation was found to be the treatment of choice for the control of acute variceal bleeding and prevention of re-bleeding with less complications.
\end{abstract}

Keywords: Band ligation, Esophageal varices

1. Assistant Professor, Department of Pediatric Gastroenterology, Hepatology \& Nutrition, Bangladesh Institute of Child Health, Dhaka Shishu (Children) Hospital.

2. Professor \& Head, Department of Pediatric Gastroenterology, Hepatology \& Nutrition, Bangladesh Institute of Child Health, Dhaka Shishu (Children) Hospital.

3. Associate Professor, Institute of Statistical Research and Training, University of Dhaka.

4. Registrar, Neonatology, Apollo Hospital, Dhaka.

5. Resident Medical Officer, Dhaka Shishu (Children) Hospital. Correspondence to: Dr. Salahuddin Mahmud, Assistant Professor, Dhaka Shishu (Children) Hospital, Dhaka. Email: drsmbablu@gmail.com

\section{Introduction}

Variceal bleeding is the most common cause of severe gastrointestinal bleeding in childhood and has significant mortality. ${ }^{1}$ Endoscopic sclerotherapy (EST) is an effective treatment for bleeding esophageal varices. However, EST is associated with substantial complications including retrosternal pain, fever, sepsis, transient dysphasia and occasionally pleural effusion. Mucosal ulcerations at the site of injection are observed in $70-80 \%$ of the patients. This is the cause 
of serious complications like rebleeding (up to $20 \%$ ), esophageal stricture and perforation. ${ }^{2,3}$ Stiegmann and Goff developed endoscopic variceal ligation (EVL) as an alternative to endoscopic sclerotherapy. ${ }^{4}$ In adult practice, variceal ligation has been shown to be safer and more effective than sclerotherapy and is now the preferred endoscopic treatment for variceal hemorrhage in adults. ${ }^{5-7}$ There is paucity of data regarding use of EVL with multi band ligator in children with variceal bleed. ${ }^{8}$ The multiband ligator has made variceal ligation easier and more comfortable for the patient. With this technique, up to 6 varices can be ligated after a single insertion of the endoscope. ${ }^{9}$ Therefore, the aim of the current study was to assess the overall outcome of band ligation in esophageal varices in terms of their eradication, recurrence, re-bleeding, safety and complications. ${ }^{10}$

\section{Materials and Methods}

A prospective study was done in the department of Pediatric Gastroenterology, Hepatology \& Nutrition, Dhaka Shishu (Children) Hospital, Dhaka, Bangladesh from April 2014 to March 2016. A total of 40 admitted cases of esophageal varices between 2-16 years of age was enrolled in this study after obtaining their consent. The etiology of esophageal varices were identified on the basis of clinical, biochemical and radiological features. Liver biopsy was done whenever it was feasible. ${ }^{8}$ Endoscopy was done after proper resuscitation by using forward viewing flexible video endoscope (Olympus GIF-V) under conscious sedation with intramuscular midazolam $(0.3 \mathrm{mg} / \mathrm{kg} /$ dose). Varices were graded on the scale based on Conn's criteria. When varices are visible only during inspiration (Grade-I), visible during both inspiration \& expiration (Grade-II), occupy $<50 \%$ of lumen (GradeIII) and $\geq 50 \%$ of lumen (Grade-IV). ${ }^{11}$

$E V L$ was done in grade II esophageal varices with red sign, grade III and grade IV in a case of acute variceal bleeding or recurrence of esophageal varices after banding. ${ }^{12}$ Multi-Band Ligator (MBL-6-F, Wilson-Cook Medical, USA) was used for EVL operation. ${ }^{13}$ Selected severe esophageal varices were visualised and aspirated into the banding chamber of the ligator. Suction was maintained until the screen became red, and then the band was deployed by rotating the handle clockwise until the band release was felt. The bands were then launched onto the selected varices in ascending order through the esophagus. ${ }^{12}$ The above procedures could been seen in Figure 1. EVL sessions were repeated at interval of 1 month till either all the varices were obliterated or reduced to grade I or tiny thrombosed varices which could not be ligated. ${ }^{8}$ During each sessions, up to 4 band were placed beginning in the distal esophagus using a multiband ligation device (six shooter). Follow up endoscopy was done every 1 month interval or when patient develop upper gastrointestinal bleeding up to minimum one year. All children were treated with band ligation plus tab. propranolol with an oral proton pump inhibitor (PPI) (Omeprazole $1 \mathrm{mg} / \mathrm{kg} /$ day) for 2 months or more until succeeding endoscopy. ${ }^{14}$ Treatment was analyzed by grading of varices, total number of band ligation sessions \& number of band required in every session, total number of obliteration, incidence of early \& late re-bleeding and complications. ${ }^{8}$ Initial variceal obliteration was defined as the complete disappearance of esophageal varices or when the sizes of esophageal varices were to small to be ligated. Recurrence of esophageal varices was defined as a re-appearance of esophageal varices or enlargement of previous small-size varices that became accessible by EVL. ${ }^{10}$ Early re-bleeding defined by recurrent hematemesis and/or malena and/or bloody fluid drained by nasogastric tube or needed blood transfusion within 1 month of band ligation \& late rebleeding defined by same manifestations $\geq 1$ months of band ligation. ${ }^{12}$

A preformed semi structured data collecting form was used as a data collection instrument. Data were collected by researcher and analyzed by Statistical Package of Social Science (SPSS) version 11.5 programme. Descriptive analysis was performed for demographic, clinical and radiographic features, and results were presented as mean \pm standard deviation for quantitative variables and frequencies (percentages) for qualitative variables. Data was analyzed by $Z$ test for proportion testing. An upper tailed $p$ value of $<0.05$ was considered as significant.

\section{Results}

Initially, 49 children with esophageal varices were enrolled, $8(16.32 \%)$ were lost from follow up of various reasons and $1(2.0 \%)$ was dead due to variceal bleeding. The final sample size was 40 . All the children were between 2-12 years of age. Subjects mean age was $7.2 \pm 4.3$ years. There were $30(60 \%)$ male \& 16 $(40 \%)$ female cases. Male: Female ratio was 1.5:1.

Out of 40 children, 32 (80\%) developed portal hypertension due to pre-hepatic causes and $08(20 \%)$ 
due to hepatic causes. No patient was found with post-hepatic cause. The mean age at onset of the first variceal bleeding was at $5.3 \pm 4.5$ years. In prehepatic cases it was $4.4 \pm 3.6$ years \& in hepatic cases it was $7.6 \pm 4.6$ years. So, in pre-hepatic cases first variceal bleed occurs much earlier than hepatic cases.

Table-I

Baseline characteristics

\begin{tabular}{lcc}
\hline Variables & Value & \\
\hline Mean Age (years) & $7.2 \pm 4.3$ & \\
Sex, n (\%) & & \\
Male & $30(60)$ & \\
Female & $16(40)$ & \\
Mean age of first variceal & & \\
bleed (years) & & \\
All cases & $5.3 \pm 4.5$ & \\
Pre-hepatic & $4.4 \pm 3.6$ & \\
Hepatic & $7.6 \pm 4.6$ & \\
\hline Etiology, n (\%) & Pre-hepatic & Hepatic \\
\hline Overall & $32(80)$ & $08(20)$ \\
Portal vein thrombosis & $20(62.5)$ & - \\
Splenic vein thrombosis & $4(12.5)$ & - \\
Others & $8(25)$ & - \\
Cryptogenic & - & $4(50)$ \\
Wilson's disease & - & $1(12.5)$ \\
Hepatistis B virus & - & $1(12.5)$ \\
Biliary cirrhosis & - & $1(12.5)$ \\
Autoimmune hepatitis & - & $1(12.5)$ \\
\hline Esophageal Varices, $\mathrm{n}(\%)$ & Pre-hepatic & Hepatic \\
\hline Grade-II with red sign & $16(50)$ & - \\
Grade-III & $08(25)$ & $04(50)$ \\
Grade-IV & $08(25)$ & $04(50)$ \\
\hline Gastric Varices, $\mathrm{n}(\%)$ & Pre-hepatic & Hepatic \\
\hline & $04(12.5)$ & $4(50)$ \\
\hline
\end{tabular}

Patients were followed up for a minimum period of one year after the band ligation. Initial variceal obliteration was achieved in all $(100 \%)$ cases.
Only one session required in $50 \%$ cases of prehepatic children and multiple session required in hepatic children. Almost same number of band required in every session of both pre-hepatic \& hepatic cases. Recurrence of bleeding was observed in $50 \%$ cases of pre-hepatic \& all cases of hepatic children. Early re-bleeding was more common in hepatic $(75 \%)$ cases \& late re-bleeding was more common in both pre-hepatic $(43.75 \%)$ \& hepatic $(100 \%)$ cases.

Ten (25\%) children experienced minor complications like discomfort $(10 \%)$, nausea $(10 \%)$ \& retrosternal pain $(05 \%)$, while major complications were absent.

Table-II

Outcome of band ligation

\begin{tabular}{lcc}
\hline Outcome & Value & \\
\hline Number of band ligation & Pre-hepatic & Hepatic \\
session required, $\mathrm{n}(\%)$ & & \\
1 session & $16(50)$ & - \\
2 session & $12(37.5)$ & $04(50)$ \\
3 session & $04(12.5)$ & $04(50)$ \\
\hline Number of band required in & Pre-heptic & Hepatic \\
\hline every session, $\mathrm{n}$ (average) & $1-3(2)$ & $1-4(2.5)$ \\
Initial obliteration, & Pre-hepatic & Hepatic \\
$\mathrm{n}$ (\%) & $32(100)$ & $08(100)$ \\
\hline Recurrence of bleeding, $\mathrm{n}(\%)$ & Pre-hepatic & Hepatic \\
\hline Overall & $16(50)$ & $08(100)$ \\
Early re-bleeding & $02(6.2)$ & $06(75)$ \\
Late re-bleeding & $14(43.8)$ & $08(100)$ \\
\hline Complications, $\mathrm{n}(\%)$ & Minor events & Major \\
events & & \\
\hline Discomfort & - & - \\
Nausea & - & $04(10)$ \\
Retrosternal pain & $04(10)$ & - \\
Esophageal ulceration & $02(5)$ & - \\
Esophageal perforation & - & 00 \\
Esophageal stricture & - & 00 \\
Aspiration pneumonia & - & 00 \\
Sepsis & - & \\
\hline & & \\
\hline
\end{tabular}



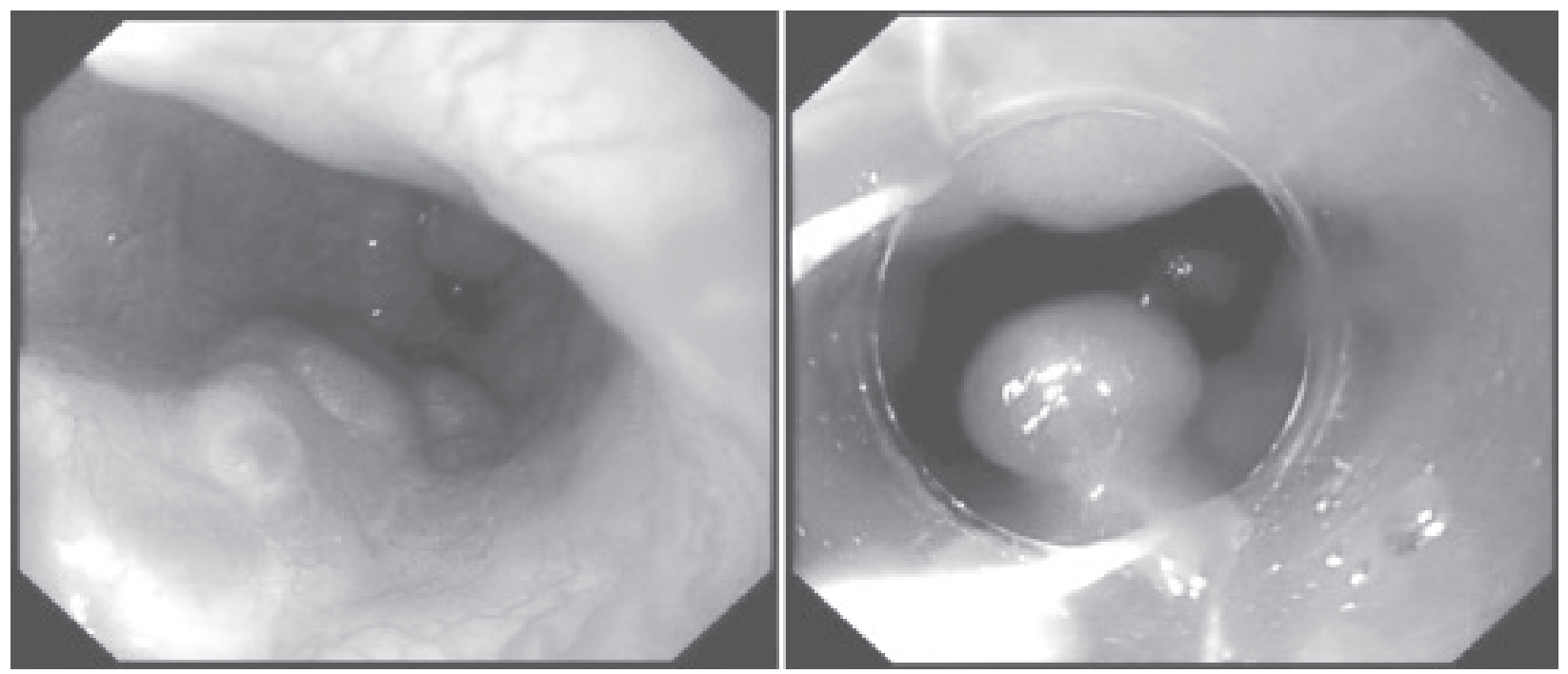

Fig.1: Endoscopic image showing large esophageal varix \& ligating the varix using multiband ligator

\section{Discussion}

Esophageal varices are the most common complication of EHPVO and IHPVO. Once varices have developed, they increases in size and eventually cause variceal bleeding. ${ }^{15}$ This is an emergency with high rates of morbidity \& mortality. ${ }^{12}$

EVL is the gold standard for the treatment of acute bleeding of esophageal varices. Furthermore, it is also effective in secondary prophylaxis and in primary prophylaxis for patients who are not suitable candidates for beta blocking agents. ${ }^{10}$ Several previous experience suggested that this technique is safe \& effective in children. ${ }^{16-20}$

A pediatric stud analyzed the endoscopic sclerotherapy \& band ligation and reached the conclusion that EVL was much more effective than scerotherapy and achieved variceal obliteration faster with fewer treatment sessions. ${ }^{10}$ In a recent randomized controlled trial comparing EST and EVL for bleeding esophageal varices in children with EHPVO, it was found that, EVL eradicated varices within fewer endoscopy sessions ( $3.9 \pm 1$ vis $6.1 \pm$ 1.7). ${ }^{13}$ In the present study, only one session required in $50 \%$ cases of EHPVO and multiple session required in IHPVO.

Multiband ligator has been used in children in few studies only. ${ }^{13,21}$ Kerner et al ${ }^{21}$ found that by using multi band ligator, varices were obliterated in 2 sessions in 26 of 28 patients with minimal complications. In these studies multi band ligator was found to be technically feasible and safe in children. Similar observation was also reported in the present study. We applied 2-3 band in every session of ligation in both pre-hepatic $\&$ hepatic cases.

In adult \& children, endoscopic therapy for variceal bleeding with EVL is a well established treatment that achieves $70 \%$ to $100 \%$ early haemostasis. ${ }^{12}$ Abbasi et $\mathrm{al}^{10}$ stated that initial variceal obliteration was achieved in $79.8 \%$ cases. In the present study, initial variceal obliteration occurred in all cases $(100 \%)$ with proper banding.

Meta-analyses of 15 studies that compared EVL against sclerotherapy in the prevention of variceal rebleeding showed that EVL was associated with lower rate of re-bleeding. ${ }^{22}$ Pokhrana et al ${ }^{8}$ also stated the same result. Recurrence of bleeding was much more in intra-hepatic cases $(91.7 \%)$ than extra-hepatic cases $(8.3 \%) .{ }^{15}$ In our study, recurrence of bleeding occurred in $100 \%$ intra-hepatic cases and $50 \%$ prehepatic cases. Masalaite et al ${ }^{15}$ also showed that early re-bleeding was more in intra-hepatic cases $(82.1 \%)$ \& less in pre-hepatic (17.9\%) cases. Similar result was observed in our study. Early re-bleeding occurred in $75 \%$ intra-hepatic cases and $6.2 \%$ prehepatic cases. Late re-bleeding was more in both prehepatic $(43.8 \%)$ \& hepatic $(100 \%)$ children.

Major complications like esophageal perforation, bleeding, ulceration and stricture formation was significantly higher in EST group as compared to EVL group. ${ }^{8}$ In a study of 17 children with EHPVO reported 
no short term or long term complication with EVL. Lu et $\mathrm{al}^{12}$ also did not observe fatal complications following EVL. We reported some minimal events like discomfort (10\%), nausea (10\%) \& retrosternal pain $(5 \%)$ but major events were absent.

\section{Conclusions}

Band ligation eradicates esophageal varices with less complications and lower re-bleeding rate. Only few sessions were required in pre-hepatic cases than in hepatic cases. Recurrence of bleeding including early re-bleeding were more common in hepatic cases. Endoscopic variceal ligation using multiband ligator was found to be safe \& effective for the control of acute variceal bleeding and the prevention of re-bleeding in children.

\section{Limitations of study}

There were mainly two limitations of our study. First, it was a single-centre study with a limited sample size and short period of follow-up (around one year). Data needs further evaluation on a larger scale and over a longer follow-up duration. Second, it was a singlearm study (only the outcome of band ligation) and so not comparable to other available options for oesophageal varices like oral drugs, endoscopic sclerotherapy or their combination.

\section{References}

1. Patrick JM, Susan VB, Suzanne MD. A prospective study of endoscopic esophageal variceal ligation using a multiband ligator. J Pediatr Gastroenterol Nutr. 2002; 34: 207-11.

2. Goncalves ME, Cardosos SR, Maksoud JG. Prophylactic scerotherapy in children with esophageal varices: Long term result of a controlled prospective randomized controlled trial. J Pediatr Surg. 2000; 35: 401-05.

3. Howard ER, Stringer MD, Mowat AP. Assessment of injection sclerotherapy in management of 152 children with esophageal varices. Br J Surg. 1988; 75: 404-08.

4. Stiegmann GV, Goff JS. Endoscopic esophageal varix ligation (EVL): Preliminary clinical experience. Gastrointest Endosc. 1988; 34: 113-17.

5. Laine L, Cook D. Endoscopic ligation compared with sclerotherapy for treatment of esophageal variceal bleeding. A metaanalysis. Ann Intern Med. 1995;123: 280-87.

6. De Franchis R, Primignani M. Endoscopic treatments for portal hypertension. Semin Liv Dis. 1999; 19: 439-55.

7. Jalan R, Hayes PC. UK guidelines on the management of variceal haemorrhage in cirrhotic patients. British Society of Gastroenterology. Gut. 2000; 46 (suppl 3-4): III 1-15.

8. Pokhrana RK, Kumar S, Khatri PC, Chahar CK. Endoscopic variceal ligation using multiband ligator. Indian Pediatrics. 2005; 42(1): 131-34.

9. Saeed ZA. The Saeed six-shooter: a prospective study of a new endoscopic multiple rubber-band ligator for the treatment of varices. Endoscopy. 1996; 28: 559-64.

10. Abbasi A, Bhutto AR, Bhatti KI, Mahmood K, Lal K. Outcome of band ligation in esophageal varices. J Pak Med Assoc. 2013; 63: 983-87.

11. Conn HO. Ammonia tolerance in the diagnosis of esophageal varices: A comparison of endoscopic, radiologic and biochemical techniques. J Lab Clin Med. 967; 70: 442-51.

12. Lu JP, Huang Y, Chen SY, Wu J. Endoscopic treatment of gastroesophageal varices: Efficacy and safety in children. HK J Pediatr (New Series) 2013; 18: 139-46.

13. Zargar SA, Javid G, Khan BA, Yattoo GN, Shah $\mathrm{AH}$, Gulzar GM, et al. Endoscopic ligation compared with sclerotherapy for bleeding esophageal varices in children with extrahepatic portal venous obstruction. Hepatology. 2002; 36: 666-72.

14. Li P, Zhang ST, Yu ZL. Analysis of the risk factors in early rebleeding after endoscopic variceal ligation. Zhonghua Xiaohua Neijing Zazhi. 2006; 23: 23-26.

15. Masalaite L, Valantinas J, Stanaitis J. Reccurence of esophageal varices after endoscopic band ligation: single centre experience. Acta Medica Lituanica. 2012; 19: 59-66

16. Fox VL, Carr-Locke DL, Connors PJ. Endoscopic ligation of esophageal varices in children. J Pediatr Gastroenterol Nutr. 1995; 20: 202-8. 
17. Nijhawan S, Patni T, Sharma U. Endoscopic variceal ligation in children. J Pediatr Surg. 1995; 30: 1455-56.

18. Price MR, Sartorelli KH, Karrer FM. Management of esophageal varices in children by endoscopic variceal ligation. J Pediatr Surg. 1996; 31: 1056-59.

19. Sasaki T, Hasegawa T, Nakajima K. Endoscopic variceal ligation in the management of gastroesophageal varices in postoperative biliary atresia. J Pediatr Surg. 1998; 33: 1628-32.
20. Reinoso MA, Sharp HL, Rank J. Endoscopic variceal ligation in pediatric patients with portal hypertension secondary to liver cirrhosis. Gastrointest Endosc. 1997; 46: 244-46.

21. Mckiernan PJ, Beatb SV, Davison SM. A prospective study of endoscopic esophageal variceal ligation using a multiband ligator. $\mathrm{J}$ Pediatr Gastroenterol Nutr. 2002; 34: 207-11.

22. Garcia-Pagan JC, Bosch J. Endoscopic band ligation in the treatment of portal hypertension. Nat Clin Pract Gastroenterol Hepatol. 2005; 2: 526-35. 